\title{
SPECTRAL CHARACTERISTICS OF A-TYPE STARS IN THE GALACTIC HALO
}

\section{J. CORBALLY}

Vatican Observatory Research Group, University of Arizona, Tucson AZ 85721, USA

R. O. GRAY

Department of Physics and Astronomy, Appalachian State University, Boone NC 28608, USA

\begin{abstract}
Among 67 early A-type stars at high galactic latitudes, selected to have both small $\mathrm{m}_{1}$ and large $c_{1}$ Strömgren photometric indices, we find about one third to have quite normal dwarf or subgiant spectra at MK classification dispersion. Those stars with peculiarities included 10 new $\lambda$ Bootis stars, though none of these appeared significantly evolved. We wonder whether some of the metal-weak dwarf stars in our set may be field horizontal-branch equivalents of the blue, downward-curving, lowluminosity end of the blue horizontal-branch stars.
\end{abstract}

\section{INTRODUCTION}

Field horizontal-branch (FHB) candidates have been listed by Philip (1984), who used thin prism spectra to find A-type stars at high galactic latitudes and then applied the photometric criteria of small $\mathrm{m}_{1}$ and large $c_{1}$ Strömgren indices to narrow the selection to FHB candidates. Since these photometric characteristics should also fit an evolved- $\lambda$-Bootis star, if such exists, we became interested in what the spectra of these stars might look like. (The $\lambda$ Bootis stars are a class of apparently metal-weak, population I, A-type stars.)

\section{RESULTS AND DISCUSSION}

Spectral Characteristics

Tentative results based on reticon spectra were presented two years ago (Corbally and Gray 1990). We have now obtained high S/N CCD spectra from the Steward Observatory $2.3 \mathrm{~m}$ telescope for 60 of Philip's FHB candidates and we have the lower quality reticon spectra for another 7 of these stars. All 67 have been homogeneously classified on the MK system. Twenty-one looked like normal dwarfs and subgiants, while the rest showed peculiarities characteristic of protoshell, field blue straggler, $\lambda$ Bootis, silicon Ap, and metal-weak stars. We were surprised at the large number (10) of $\lambda$ Bootis-type stars, one of which has 
been identified in the literature as a prototype FHB star (Fig. 1), and at the paucity (only 2) of low gravity stars among what are FHB candidates with a large $c_{1}$ index. Regarding the original motivation for this study, we concluded, after a reconsideration based on the improved spectral data, that even with the two best candidates (Fig. 2) we had not found a significantly evolved- $\lambda$-Bootis star.

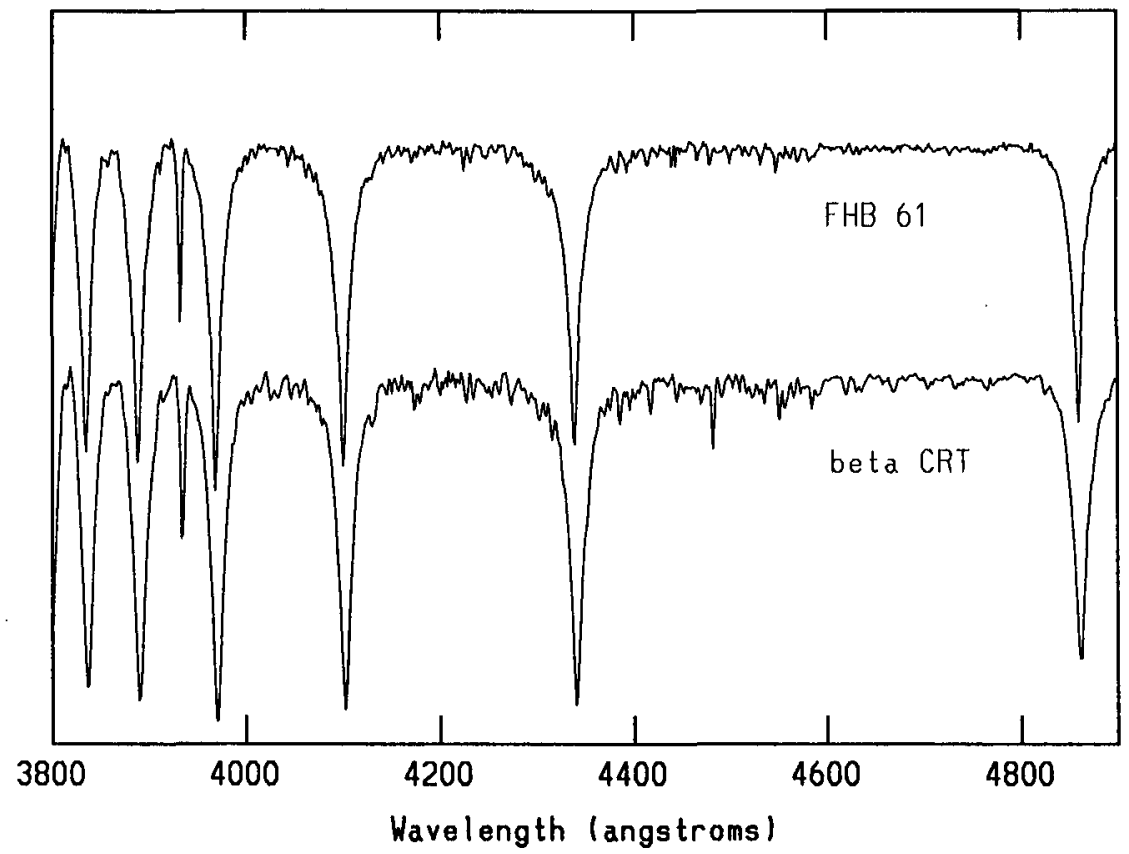

Fig. 1. FHB 61 is HD 2857 and one of four prototype FHB stars (Oke, Greenstein and Gunn 1966); $\beta$ Crt is a standard A2 IV star. A $\lambda$ Bootis star can be distinguished from a normal star in this range of wavelengths by the general weakness of the metal lines, the extra weakness of $\mathrm{Mg}$ II 4481 (see especially the ratio $\mathrm{Mg}$ II $4481 / \mathrm{Fe}$ I 4383) and by the slightly peculiar hydrogen lines with narrow, weak cores, but broad wings. We classified FHB 61 as A2 IV $\lambda$ Boo (PHL) since the peculiarity of its hydrogen lines is not marked.

The $\lambda$ Bootis Stars

Since the ten new $\lambda$ Bootis stars have distances from the galactic plane as much as $4.5 \mathrm{kpc}$, our results raise questions for the accretion-diffusion theory of the origin of $\lambda$ Bootis stars (Charbonneau 1991). Questions also arise about their evolutionary status and about their relationship to Population II stars. Answers may strengthen the link between $\lambda$ Bootis stars and post-AGB stars (Lambert 1991), but this would seem to contrast with their occurrence among young, nearZAMS stars (Gray and Corbally 1992). However, Parthasarathy (1992) has suggested that processes in circumstellar dust shells may produce the similar 
abundance phenomena found in both pre-main sequence and post- AGB stars.

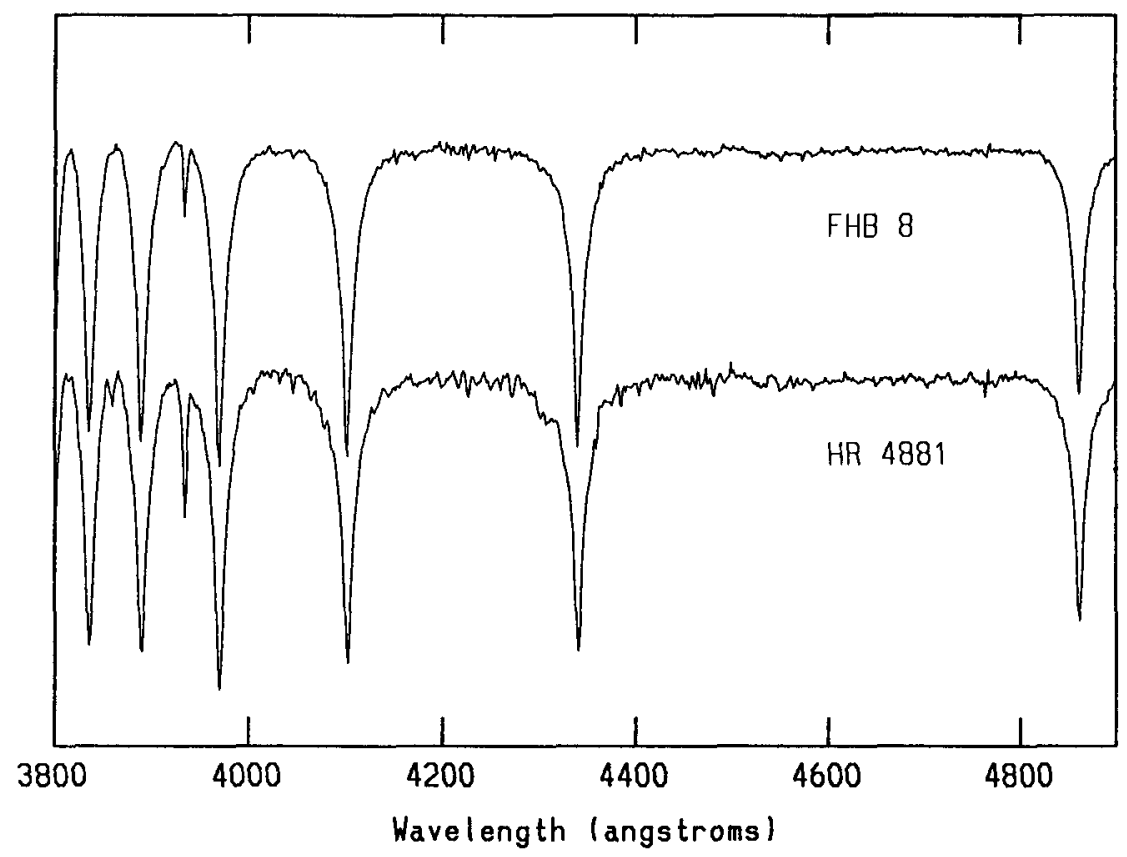

Fig. 2. CCD spectra of FHB 8, a star whose reticon spectrum suggested it to be an evolved- $\lambda$-Bootis star, and HR 4881, a "standard" $\lambda$ Bootis star typed as A1.5 Va- $\lambda$ Boo PHL by Gray (1988). In FHB 8 the broad wings of its peculiar hydrogen lines suggest that it is a dwarf $\lambda$ Bootis star like HR 4881 and that it has a similar effective temperature. However, there is an extreme weakness of the metal lines for which simply calling FHB 8 earlier in spectral type than HR 4481 is not the complete description. That extreme weakness was echoed in the red region by the $O 7774$ triplet, $\mathrm{O} 8446$, and $\mathrm{Ca}$ II 8498, 8542 lines. We classified its blue spectrum as A0 Va $\lambda$ Boo PHL, extreme metal weak. This may be a Population II analogue of the $\lambda$ Bootis stars.

The Metal- Weak Dwarfs

These results also suggest that there are two characteristics for blue horizontalbranch stars (MacConnell et al. 1971, Dworetsky 1984), even for those as late as the early A-type. One group of BHB stars have sharp Balmer lines and weak metallic lines like two in our sample. However, those from the blue, downwardcurving, low-luminosity end of the BHB may look very much like metal-weak dwarfs (Fig. 3). More spectroscopic study of those blue, lower luminosity BHB stars in globular clusters is needed to verify this tentative conclusion. 


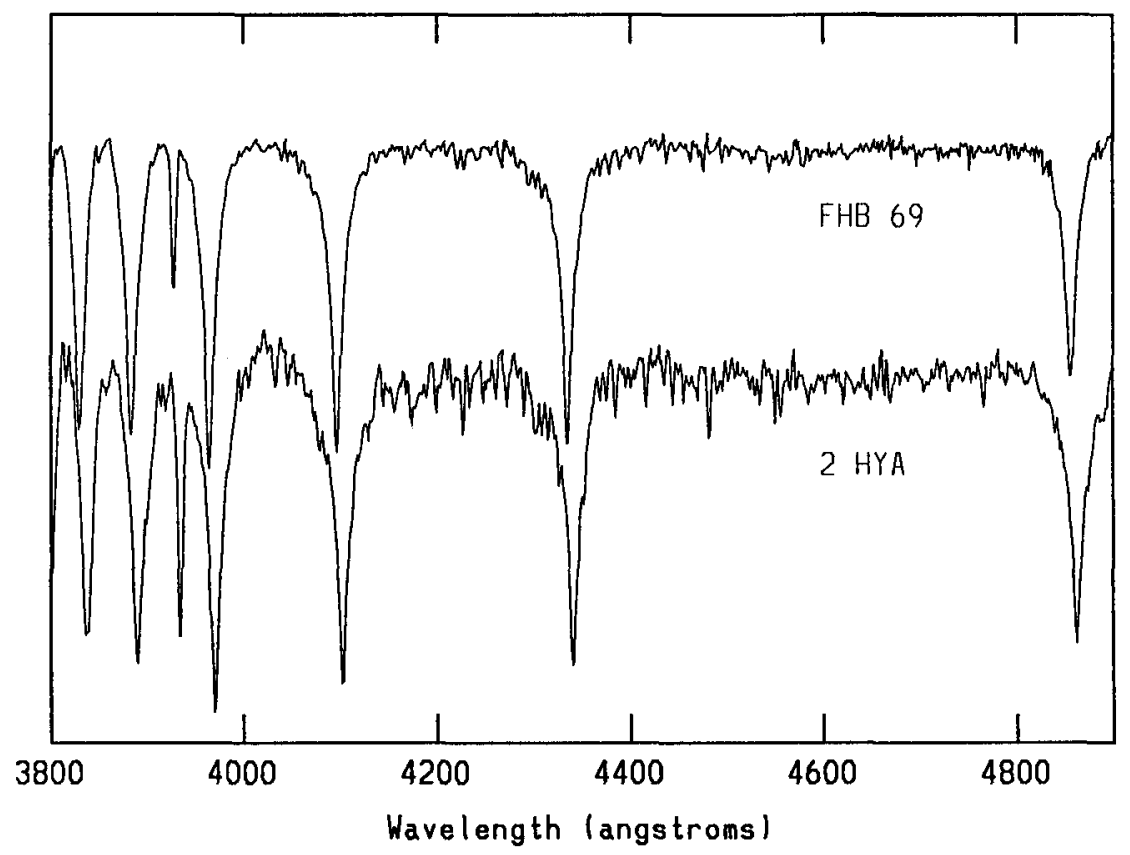

Fig. 3. FHB 69 is HD 161817 and another prototype FHB star from Oke, Greenstein and Gunn (1966). We classified it as A8 V kA2mA1 (an A8 dwarf with weak metallic lines) for, though photometry and spectrophotometry indicate a large Balmer jump for FHB 69, its hydrogen wings are as broad as the standard A7 V star, 2 Hya, shown below it. Perhaps these photometric and spectroscopic features are characteristic of stars from the blue, lower luminosity end of the horizontal branch.

\section{REFERENCES}

Charbonneau, P. 1991, ApJ, 372, L33

Corbally, C.J., and Gray, R.O. 1990, in Poster Papers Presented at the 145th Symposium of the $I A U$, ed. G. Michaud, A. Tutukov, M. Bergevin (Univ. of Montreal), p.55

Dworetsky, M. 1984, in The MK Process and Stellar Classification, ed. R.F. Garrison (David Dunlap Observatory), p.389

Gray, R.O. 1988, $A J, 95,220$

Gray, R.O., and Corbally, C.J. 1992, in IAU Coll., No. 138, Peculiar Versus Normal Phenomena in A-Type and Related Stars, ed. F. Castelli and M.M. Dworetsky

Lambert, D.L. 1991, in LAU Symp., No. 145, Evolution of Stars: The Photospheric Abundance Connection, ed. G. Michaud and A. Tutukov (Dordrecht: Kluwer), p.451

MacConnell, D.J., Frye, R.L., Bidelman, W.P., Bond, H.E. 1971, PASP, 83, 98 
Oke, J.B., Greenstein, J.L., and Gunn, J. 1966, in Stellar Evolution, ed. R.F. Stein and A.G. Cameron (New York: Plenum), p.399

Parthasarathy, M. 1992, IAU Coll., No. 138, Peculiar Versus Normal Phenomena in A-Type and Related Stars, ed. F. Castelli and M.M. Dworetsky

Philip, A.G.D. 1984, VVO Contributions, No.2, 1

\section{DISCUSSION (Corbally and Gray)}

ADELMAN: One can use rotation to separate out true FHB stars from the FHB candidates. Ruth Peterson has found that the FHB stars have maximum $v \sin i$ values of about $35 \mathrm{~km} \mathrm{~s}^{-1}$. Thus, moderate dispersion spectra can help in this regard.

CORBALLY: The resolution of our spectra, about $3 \AA$, is not sufficient to distinguish rotation as slow as $35 \mathrm{~km} \mathrm{~s}^{-1}$, but $\mathrm{I}$ thank you for this additional clue to the true FHB stars.

ADELMAN: There are also a number of halo A-type stars such as 7 Sex and HD 64486, with Pop I abundances and kinematic characteristics which make them more like Pop II. They indicate something interesting must have happened in the relatively recent history of the halo.

CORBALLY: Since the hydrogen line strength has a rather flat maximum with temperature for the early A-type stars, we can only be certain of an abundance less than about -1 dex when we call one of these FHB stars "metal weak." However, the very normal-looking FHB candidate stars not only have near Pop I abundances and the kinematics of Pop II stars, but also have the abnormal Strömgren indices, i.e., large $c_{1}$ and small $\mathrm{m}_{1}$.

DWORETSKY: Spectroscopic methods won't give a $\mathrm{T}_{\text {eff }}$ easily, but you could try using the Strömgren parameters $a$ and $r$ in the region of the Balmer line maximum. They should work well; for best results, use the parameters $a_{0}$ and $\mathrm{r}^{\star}$, and the grid of Moon and Dworetsky (MNRAS, 217, 305, 1985).

CORBALLY: This method of obtaining $T_{\text {eff }}$ is obviously important for the detailed abundance analysis which should be done on some of these "FHB" stars. COWLEY: I assume FHB 69 is a metal-weak star. I am not convinced that it makes sense to exclude it from interest because it doesn't have some arbitrarily chosen criterion for the $\lambda$ Boo class. I'm not sure this discrimination makes sense; perhaps it does.

CORBALLY: Yes, we classified FHB 69 (= HD 161817) as a metal-weak star (A8 V kA2mA1) and so as interesting in that respect and deserving of detailed analysis. However, just as, say, the Ap silicon stars are distinguishable as a class of peculiar stars on the MK system, so too the $\lambda$ Boo stars (not just weak lined, but also with especially weak Mg II $4481 \AA$ line) are a distinct class of peculiar stars and not arbitrary. These distinct classes are best studied separately, unless it subsequently appears that they are related.

HACK: I wish to remind that Feige 86 is a very peculiar halo star. It has been studied by Bonifacio, Castelli and me in the far UV. It shows strong $\mathrm{C}$ deficiency and $\mathrm{Ga}$ excess; according to Sargent et al., it is very similar to $3 \mathrm{Cen} \mathrm{A}$, at least from the optical spectrum. Do other halo peculiar stars exist?

CORBALLY: I do not know of other stars with these peculiarities. At MK classification dispersion these peculiarities would be undetectable, and so finding similar stars seems a problem. 